wie auch mit hohem Risiko. Entscheidungen, die den optimalen Entbindungstermin betreffen, sollten auch die Risiken für jene Feten berücksichtigen, die im Mutterleib verbleiben. Dr. Robert Bublak

Mandujano A et al. The risk of fetal death: current concepts of best gestational age for delivery. Am J Obstet Gynecol 2013; 208: 207.e1-8

Kommentar: Besonders im Zusammenhang mit der Wunschsectio ist in den letzten Jahren international viel über das optimale Entbindungsgestationsalter bezüglich neonataler Mortalität und Morbidität diskutiert worden. Mandujano et al. haben erstmals auch die registrierten fetalen Todesfälle und Totgeburten in ihre Betrachtungen eingeschlossen. Der allgemeine Konsensus, dass der optimale Entbindungszeitpunkt, basierend auf neonatalen Daten $39+0$ Gestationswochen ist, war die grundlegen- de Hypothese. Die Frage dabei, was ist das fetale Risiko, unentbunden zu bleiben?

Das Grundkonzept, fetale Todesfälle zu vermeiden, muss balanciert werden gegen steigende neonatale Mortalität und Morbidität. Nur die neonatale Mortalität betrachtend muss berücksichtigt werden, dass es natürlich einen Anteil neonataler Todesfälle gibt, der in keiner Weise durch Frühgeburtlichkeit bedingt ist. Eine entsprechende Basisrate von 0,5 Promille wurde angenommen und in den Kalkulationen für einen optimalen Entbindungstermin als unvermeidlich registriert. Dies berücksichtigend, d.h. Vermeidung fetaler Todesfälle und Vermeidung neonataler Todesfälle, zeigte sich, dass $37+0$ das optimale Entbindungsalter bei Schwangerschaftsrisiken ist - berücksichtigt man dieses Konzept: Minimierung der fetoneonatalen Gesamtmortalität.

Die Pitfalls der Untersuchungen werden in einer sehr subtilen, kritischen Art festgestellt,
z.B. wann ein intrauteriner Todesfall wirklich eingetreten ist und wann er festgestellt wird. Auch die Einflussgröße der regelmäßigen fetalen Überwachung, besonders bei Hochrisikoschwangerschaften, ist aus diesen $\mathrm{Da}$ ten nicht ableitbar und hat auch hier Implikationen für die Rate fetaler Todesfälle. Auch wird - das erscheint wesentlich - die neonatale Morbidität und das Langzeit-Outcome entsprechender Kinder nicht berücksichtigt.

Die unangefochtene Tatsache, dass neonatale Todesfälle ihr Minimum mit $39+0$ Wochen erreichen, muss nach dieser Analyse bezüglich der dann schon wieder steigenden fetalen Todesfälle unter Umständen relativiert werden. Insgesamt erscheint eine Herangehensweise im Sinne einer personalisierten Medizin, vor allem durch ein interdisziplinäres Gespräch, eine sinnvolle Lösung zu sein. Implikationen für den Zeitpunkt einer Wunschsectio hat diese Studie meines Erachtens nicht.

Prof. Jens Möller

\title{
CIN2-3-Läsionen nach LEEP: HPV-Impfung vermindert Rezidivrate
}

Nach Behandlung von CIN2-3 Läsionen mittels elektrochirurgischen Schlingenexzisionsverfahrens (LEEP) kommt es bei bis zu 30\% der Frauen zu einem Rezidiv. Eine aktive HPV-Impfung nach der Behandlung, senkt die Rezidivrate.

$\mathrm{D}$ as elektrochirurgische Schlingenexzisionsverfahren (LEEP) wird zur Therapie höhergradiger zervikaler intraepithelialer Neoplasien (CIN) eingesetzt. Unbehandelte CIN2-3-Läsionen, die durch onkogene humane Papillomviren (HPV) verursacht werden, können in ein Zervixkarzinom übergehen. Studien zeigten, dass es bei 5-30\% nach einer LEEP zu einem Rezidiv kommt, weswegen Nachfolgeuntersuchungen sowie eine erneute Therapie notwendig werden. Zur primären Prävention von CIN2-3 stehen zwei aktive Impfstoffe, ein bivalenter (HPV-16/18) und eine quadrivalenter (HPV-6/11/16/18), zur Verfügung, die sich in klinischen Studien als hochwirksam erwiesen haben. Eine retrospektive Studie zeigte, dass bei Frauen, die mit der quadrivalenten HPV-Vakzine geimpft wurden und bei denen eine HPV-bedingte Erkrankung operativ behandelt worden war, seltener ein Rezidiv auftrat. Eine prospektive Studie aus Korea untersuchte

jetzt die Frage, die sich im gynäkologischen Alltag häufig stellt: Sollen Frauen nach einer LEEP wegen CIN2-3 noch aktiv gegen HPV geimpft werden? In der Studie wurden Frauen $(n=737)$ im Alter von 20-45 Jahren, bei denen eine CIN2-3 Läsion mittels LEEP behandelt worden war, über 24 Monate nachverfolgt. Eine Hälfte wurde mit der quadrivalenten HPV-Vakzine geimpft, die andere Hälfte erhielt keine Impfung. Unabhängig vom verursachenden HPV-Genotyp kam es bei $36 \%(4,9 \%)$ der Frauen zu einem Rezidiv. Dabei trat bei 2,5\% der geimpften Frauen ein Rezidiv auf, dagegen erkrankten $7,2 \%$ der nicht geimpften Frauen erneut. Die Auswertung auf die Vakzinetypen (HPV-16/18) ergab, dass es bei 2,5\% der geimpften Frauen und bei 8,5\% der nicht geimpften Frauen $(p<0,01)$ zu einem Rezidiv kam. Die Multivariantenanalyse zeigte, dass keine Impfung nach der LEEP ein unabhängiger Risikofaktor (Risiko 2,84; 95\% Konfidenzintervall,
1,335-6,042; $p>0,01$ ) für das Auftreten eines Rezidivs ist. Die Autoren folgern daher, dass die HPV-Impfung nach einer LEEP erwogen werden sollte.

Fazit: Nach Krankenkassenangaben werden in Deutschland jährlich ca. 140.000 operative Maßnahmen (z.B. LEEP) wegen HPV-bedingten höhergradigen Läsionen an der Zervix durchgeführt. Mit dieser Studie wird eine wichtige Frage beantwortet, die bisher aufgrund der fehlenden Datenlage nur ,per Bauchgefühl“ beantwortet werden konnte. Die Ergebnisse belegen, dass durch eine HPV-Impfung nach einer LEEP das Risiko für ein Rezidiv insgesamt sowie bezogen auf die Vakzinetypen deutlich gesenkt werden kann. Aufgrund der unverständlichen schlechten Akzeptanz der prophylaktischen HPV-Impfung von Teenagern in Deutschland wird es auch weiterhin zu CIN2-3 Läsionen kommen. Daher sollten wenigstens Frauen nach einer LEEP durch die Impfung vor einem Rezidiv geschützt werden.

Prof. Tino F. Schwarz

Kang WD et al. Is vaccination with quadrivalent HPV vaccine after loop electrosurgical excision procedure effective in preventing recurrence in patients with high-grade cervical intraepithelial neoplasia (CIN2-3)? Gynecol Oncol 2013 http:// dx.doi.org/10.1016/j.ygyno.2013.04.050 